\title{
Economics
}

The Open-Access, Open-Assessment E-Journal

Vol. 12, 2018-20 | April 23, 2018| http://dx.doi.org/10.5018/economics-ejournal.ja.2018-20

\section{Learning to forecast, risk aversion, and microstructural aspects of financial stability}

\author{
Alessio Emanuele Biondo
}

\begin{abstract}
This paper presents a simulative model of a financial market, based on a fully operating order book with limit and market orders. The heterogeneity of traders is characterized not only with regards to their trading rules, but also by introducing a behavioral individual risk aversion and a learning ability influencing the process of expectations formation. Results show that individual learning may play a role in stabilizing the aggregate market dynamics, whereas the risk aversion has, counterintuitively, the opposite effect.
\end{abstract}

(Published in Special Issue Agent-based modelling and complexity economics )

JEL E44 E47 C63

Keywords Order book; learning to forecast; risk aversion; agent-based models

\section{Authors}

Alessio Emanuele Biondo, University of Catania, Italy, ae.biondo@unict.it

Citation Alessio Emanuele Biondo (2018). Learning to forecast, risk aversion, and microstructural aspects of financial stability. Economics: The Open-Access, OpenAssessment E-Journal, 12 (2018-20): 1-21.

http://dx.doi.org/10.5018/economics-ejournal.ja.2018-20 


\section{Introduction}

Macroeconomics reveals to be an expression of complexity, as discussed in Kirman (2010) and Ladyman et al. (2013). Although several definitions of complex systems are available, it can be synthetically said, as in Gallegati and Richiardi (2009), that a complex system is characterized by the notion of emergence, i.e., the spontaneous formation of self-organized structures at a different layers of a hierarchical configuration. In a complex system, then, the interaction among several individual parts generates aggregate outcomes which qualitatively differ from the features of its constituents. One of the most dramatic consequence of this, as explained by Prigogine (1997), is that any prediction about the timing of such emergent properties is only a waste of time. The theoretical consequences of complexity in macroeconomics are substantial, both in terms of research perspectives and of modeling tools, as shown in relevant literature, Gatti et al. (2011), Tesfatsion (2006), Hommes (2001, 2006).

From a policy perspective, the recognition of such a complex nature of aggregate Economics should induce to ground the policy design very differently. Indeed, important suggestions may arise from the analysis of the effects produced by different individual characteristics on the global dynamics. An example of such an approach can be found in Biondo (2018a,b), where some microstructural properties of the order book mechanism are studied in order to provide intuitions on possible policies for market stabilization.

The motivation of this paper is to check the effects due to heterogenous risk aversion and adaptive learning ability of traders on the stability of a financial market that operates through a realistic order book. Thus, the main result is the provision of the macro effects of micro features, which cannot act directly on the aggregate outcome but have a role in influencing the interactions among individuals. As held by Mitchell (2009), the order book is a valid example of a complex system because its dynamics emerges as a global result of local individual interactions among traders. Thus, this paper makes a step forward in the direction of investigating how global extreme events, which characterize the behavior of actual markets, are possibly determined by individual characteristics of market participants determining how their orders are eventually managed and negotiated.

A vast literature on financial order book modeling exists. Crucial surveys can be found in Chakraborti et al. (2011), Slanina (2008), and Parlour and Seppi (2008). Some existing models can be labelled as trader-centric, because they have been mainly based on frameworks aiming to derive fully rational optimal trading strategies, as in Chakravarty and Holden (1995), Foucault (1999), Parlour (1998), Hollifield et al. (2004), Hollifield et al. (2006), Roşu (2009), Rosu (2016); some other contributions can be named facts-centric, because they tended to study more the statistical features of the market as a dynamic process than the individual characterization of investors, as in Bak et al. (1997), Maslov (2000), Daniels et al. (2003), Farmer et al. (2005), Bouchaud et al. (2009), Cont et al. (2010).

This paper is methodologically linked to a third stream of literature, which is inspired by the computational approach of agent-based models (ABMs) in economics. Such models, developed since the Nineties, have shown to be able to describe many aspects neglected by the orthodox modeling, as explained in Tesfatsion (2006). Examples are, among others, Brock and Hommes (1997), Brock and Hommes (1998), Chiarella (1992), Chiarella et al. (2001), Day and Huang (1990), Franke and Sethi (1998), Hommes (2001), Lux (1995, 1998), Lux and Marchesi (1999). The heterogeneity of individuals and the global properties emerging from their interaction can be analyzed by means of specific statistical tools, as shown in Mantegna and Stanley (1999), and assumes a determinant descriptive role in models of financial markets, as in Hommes (2006), LeBaron (2006), and in models of order books, as in Raberto et al 
(2001), Chiarella and Iori (2002), Consiglio et al. (2005), Gil-Bazo et al. (2007), Chiarella et al. (2009), Tedeschi et al. (2012).

In some recent works, the individual risk aversion has been considered in agent-based models to differentiate individual behavior. In particular, Silvestre (2017) adopts a model in which the probability of default of countries is explained by means of a modified version of Tirole (2015), in which the risk aversion is negatively correlated with both default probability and income, whereas Epstein (2002) describes different evolution of civil violence among citizens with different risk perceptions, and Haer et al. (2017) show implications on flood risk analysis of different individual risk aversion of households. Focused on the description of financial markets, Fabretti and Herzel (2017) discuss the effects of convex incentives on trading behavior of agents in a naive rational-investors-vs-noise-traders model, by showing that the risk aversion (through the incentives) may affect market dynamics. A broader approach is used by Aliabadi et al. (2017), who show how agents' behavior varies according to the combined effect of individual risk attributes and to learning abilities to account for errors done in past predictions. Other recent contributions show the impact of self-correcting behavior on long-run expectations, as in Colasante et al. (2018). In Colasante et al. (2017) an experiment is shown to report that agents use adaptive expectations instead of rational ones and that this may lead to a form of collective rationality (despite the absence of communication among participants), which consists in a robust divergence of predictions from the fundamental value. In particular, as in Colasante et al. (2015), traders systematically end up with an underestimation of the fundamental price. In Bao et al. (2017) the origin of bubbles and crashes is questioned in terms of prediction errors: presented results of experiments in which non-optimal trend-following behaviors synchronize and reinforce the positive feedbacks between expectations and realised prices, even in presence of high trading heterogeneity. This exacerbates price oscillations and let optimism and pessimism arise even in presence of a stable fundamental value. In Hommes and Lux (2013) it is shown that by fitting a genetic algorithms model of learning to laboratory experiments it is possible to get fruitful intuitions on market features for suitable policies.

The agent-based model here presented enriches the existing literature on the topic with regards to several aspects. First of all, heterogeneity is modeled not only in terms of behavioral attitude (usually distinguished between fundamentalists and chartists) and with respect to individual informative sets (also within groups), but also in terms of risk aversion (by means of individual risk profiles) and adaptive learning ability (by means of the personal capability to remember past forecasting errors). Secondly, orders (which can have variable quantity) have a time validity and they can be canceled before execution. Third, the double auction mechanism governing the order-book results in a true contracts-driven price formation, in such a way that the simulated price series is entirely generated by the model and never added by any fictitious data, differently from Chiarella and Iori (2002) and Chiarella et al. (2009), among others. Fourth, the quantity management system, designed for market orders, realistically executes negotiations involving more counterparts when ordered quantities do not match.

The paper is organized as follows: section two contains the model description and validates the proposed framework by showing its compliance with the most acknowledged stylized facts of true financial data; section three is dedicated to simulations addressing the role played by individual features on market stability; section four will conclude. 


\section{The Market}

Consider a fully connected network of heterogeneous agents as in Biondo (2018a). In analogy with a wide body of existing literature, traders are distinguished according to their approach to information in considering the asset being observed. Two groups are formed, as in Kyle (1985), Copeland and Galai (1983), Glosten and Milgrom (1985), Glosten (1994), among others: the first one is constituted by fundamentalists, who form their opinions by looking at information regarding the economic activity of the issuer and presume to know the "correct" value for the asset, i.e., the fundamental value; the second one is formed by chartists, who refer to the past asset prices and estimate a reference value in order to infer the future trend. It is worth noticing that such a classification highlights two opposite behavioral attitudes in selecting information to be used: indeed, fundamentalists rely on exogenous signals coming from outside the market dynamics, whereas chartists depend completely on past market dynamics, i.e., endogenous signals. The model accounts also for a variable accuracy of the owned information within each group. This results in truly heterogenoeus trading behaviors, as it will be explained below.

\subsection{Model Description}

The differentiation of traders is further detailed by giving each market participant a personal coefficient of risk aversion and an individual possibility to learn from the past. Such features should be read in terms of informative qualifications of agents, in order to consider the consciousness of investors with regards to both the past and the future. The execution of orders depends on a double auction mechanism, which relies on an order book where investors posts their orders (both market and limit orders), according to their heterogenous expectations. The effective registration of orders and the accountancy of negotiations are operated by a realistic order book without transaction costs. The series of simulative steps, from the expectations formation to the final execution of transactions, is marked by a sequence of phases within each cycle-run of the model. Such a sequence is preceded just by the order-validity management routine, which identifies those traders who will actively participate to the successive steps and let the others (with active orders still pending in the order book) inactive for the current round. The steps are: 1) the expectations setting; 2) the strategy setting; 3) the order setting; 4) the order book management; 5) the trading. After each transaction, the price used for the negotiation is registered and a new cycle-run starts.

\section{Expectations setting}

The very first step of the individual decision process starts from the individual expectation on the price dynamics. Fundamentalists presume to know the so-called fundamental value, i.e., the long-run correct value of the asset, at each time step, $F V_{t}$. The fundamental value is an exogenous variable, whose dynamics, following Biondo et al. (2017), is set by

$$
F V_{t}=F V_{t-1}+\mathbb{D}_{t}
$$

where $\mathbb{D}_{t}$ is a bounded random variable, drawn with uniform distribution within the interval $\left[-\sigma_{\mathbb{D}}, \sigma_{\mathbb{D}}\right]$ and $F V_{0}$ is set at the beginning of simulations. In such a way, the model provides a basic representation of the yield of the asset (which is assumed to follow a random walk), by interpreting the fundamental dynamics as guided by the dividend value, which could be either positive or negative (in case of profits or losses, respectively). However, each fundamentalist has her individual perception, possibly imperfect of $F V_{t}$. Eventually, the price expectation of 
the fundamentalist $i$ at time $t$ is computed as:

$$
{ }_{F}^{i} p_{t}^{\exp }=F V_{t} \pm \Phi_{t}
$$

where $\Phi_{t}$ is a bounded random variable, with uniform distribution in $\left[-\sigma_{F}, \sigma_{F}\right]$. The individual price forecast of a chartist is, instead, built upon the inspection of past prices. Similarly to the approach adopted in Alfi et al. (2008), each chartist considers a portion of the past market prices series, within a time window whose length is individually chosen, in order to define her personal reference value, which is computed as:

$$
{ }^{i} R V_{t}=\sum_{j=(t-\tau)}^{t} p_{j} / \tau
$$

By Eq. 3 the average of last $\tau=\left(\tau_{0}+\tau_{i}\right)$ prices, included in the chosen observational time window is computed, where $\tau_{0}=200$ and $\tau_{i}$ is an individual random variable with uniform distribution, in $(0, \delta)$. Then, the individual expected price of chartist $i$ is:

$$
{ }_{C}^{i} p_{t}^{\exp }=p_{t}+\frac{p_{t}-{ }^{i} R V_{t}}{\tau-1} \pm \Lambda_{t}
$$

where $p_{t}$ is the current market price and $\Lambda_{t}$ is a uniformly distributed random variable bounded in $\left[-\sigma_{C}, \sigma_{C}\right]$, which ensures the possibility of heterogeneous expectations even among traders with same time-window lengths.

Both eqs.(2) and (4) are widely acknowledged in related literature. Similar approaches can be found in Lux and Marchesi (1999), Takayasu et al. (2006), Alfi et al. (2006, 2007). In terms of the composition of the community of agents, this configuration is conceptually different from the one used in other contributions, as Chiarella and Iori (2002) and Chiarella et al. (2009), where each trader was designed as a weighted average of three components fundamentalist, chartist, and noisy, with weights drawn from Gaussian distributions.

\section{Strategy setting}

The trading strategy, for any trader type ${ }_{j}=F, C$, is set according to the following simple rule:

- if trader $i$ expects a future price greater than the current one, i.e. ${ }_{j}^{i} p_{t}^{\exp }>p_{t}$, she will post a bid order and she will be a bidder, $B_{i}$;

- if trader $i$ expects a future price smaller than the current one, i.e. ${ }_{j}^{i} p_{t}^{\exp }<p_{t}$ she will post an ask order and she will be an asker, $A_{i}$;

- in case of stationary expectations, i.e. ${ }_{j}^{i} p_{t}^{\exp }=p_{t}$, the trader will be a holder, and the trading strategy will be neither buy, nor sell.

\section{Order setting}

In this step, the price and the quantity of the order are decided. For bid orders, the price is defined according to the willingness to pay of the bidder, as a function of expectations:

$$
{ }_{B}^{i} w_{t}={ }^{i} p_{t}^{\exp }-{ }_{A}^{i} w_{t}^{\exp }
$$

where ${ }^{i} p_{t}^{\text {exp }}$ is the expected price of $i$ and ${ }_{A}^{i} w_{t}^{\exp }$ is $i$ 's individual opinion about the askers' willingness to accept. As in Biondo (2018a), such an opinion is calculated by reverting the expectation, i.e. by assessing the hypothetical willingness to accept that the bidder would 
have expressed if she had been an asker instead, ${ }^{i} p_{t}^{\text {rev }}=p_{t}-\left({ }^{i} p_{t}^{\text {exp }}-p_{t}\right)=2 p_{t}-{ }^{i} p_{t}^{\text {exp }}$, and then by weighting it by means of a random parameter ${ }^{i} \mu_{\alpha}$ drawn, with uniform distribution, in $\left(0, \sigma_{\mu_{\alpha}}\right)$. Thus, finally, ${ }_{A}^{i} w_{t}^{\exp }={ }^{i} \mu_{\alpha}{ }^{i} p_{t}^{\text {rev }}$.

The model follows the approach of Lux and Marchesi $(1999,2000)$, which suggests that traders are able to perceive the market pressure deriving from demand/supply mismatch, $\Delta n=f\left(n_{A}, n_{B}\right)$, with $n_{A}$ and $n_{B}$ being, respectively, the number of askers and the number of bidders. Then, the price of the bid order created by bidder $i$ is:

$$
{ }_{B}^{i} p_{t}={ }_{B}^{i} w_{t}+{ }^{i} \mu_{\beta}\left[z_{1} \Delta n+z_{2} \Delta p\right]
$$

where: $\Delta p$ is the difference between the best quotes of the order book, which are computed as explained below, and are visible to all traders, as in true markets; ${ }^{i} \mu_{\beta} \in\left(0, \sigma_{\mu_{\beta}}\right), z_{1} \in\left(0, \sigma_{z_{1}}\right)$, and $z_{2} \in\left(0, \sigma_{z_{2}}\right)$ are random variables with uniform distribution, which measure the influential weight of the market environment perceived by trader $i$. More details on $\Delta n$ and $\Delta p$ will be provided later.

The symmetric situation of an asker straightforwardly follows the same rationale. The assessment of the hypothetical willingness to accept of the agent asker, computed as a function of her expectations, leads to:

$$
{ }_{A}^{i} w_{t}={ }^{i} p_{t}^{\exp }+{ }_{B}^{i} w_{t}^{\exp }
$$

where ${ }_{B}^{i} w_{t}^{\text {exp }}={ }^{i} \mu_{\alpha}{ }^{i} p_{t}^{\text {rev }}$ is defined according to the same process above illustrated and the price of the ask order created by asker $i$ is:

$$
{ }_{A}^{i} p_{t}={ }_{A}^{i} w_{t}+{ }^{i} \mu_{\beta}\left[z_{1} \Delta n+z_{2} \Delta p\right]
$$

The quantity to be ordered is chosen by each trader $i$ according to her endowment ${ }^{i} W$, which is initially distributed to all agents, consisting in an amount of money ${ }^{i} m$ and a quantity of shares ${ }^{i} a$, and evolves in time due to negotiated transactions. The model does not allow short-sales. Each order is then referred to a feasible interval and quantity is drawn randomly from it, in order to ensure that a bidder can decide to buy, at most, the highest number of shares she can pay, and an asker can decide to sell, at most, all shares she has. For bid $\left({ }^{i} q_{t}^{B}\right)$ and ask $\left({ }^{i} q_{t}^{A}\right)$ orders, quantities will be set as:

$$
{ }^{i} q_{t}^{B}=\omega \quad \text { and } \quad{ }^{i} q_{t}^{A}=\eta
$$

where $\omega \in\left[1, m_{t} / p_{t}\right]$ and $\eta \in\left[1, a_{t}\right]$.

\section{Order book management}

Orders are then submitted to be registered in the order book. As in true markets, two sections of the book exist and they are ranked by the prices of the orders. The demand side contains all bid orders, ranked decreasingly in such a way that the highest bid price, named best bid $\left({ }_{B} p_{t}^{\text {best }}\right)$, is the first of the list, and the trader who posted it, i.e. the best bidder, has the priority (because her willingness to pay is the highest). The supply side contains all ask orders, ranked increasingly in such a way that the lowest ask price, i.e. best ask $\left({ }_{A} p_{t}^{\text {best }}\right)$, is the first of the list, and the trader who posted it, i.e. the best asker, has the priority (because her willingness to accept is the lowest). Although a wide taxonomy of orders exists in true markets, the model presents only two types of them, as in Chiarella et al. (2009): limit and market orders. The distinction relies on the comparison between their prices $\left({ }_{A} p_{t}\right.$ and $\left.{ }_{B} p_{t}\right)$ and the current counter-side best quotes. 
A- An ask order, posted by a trader $i$, will be:

- a limit order, if ${ }_{A}^{i} p_{t}>_{B} p_{t}^{\text {best }}$

- a market order, if ${ }_{A}^{i} p_{t} \leq_{B} p_{t}^{\text {best }}$

B- A bid order, posted by a trader $i$, will be:

- a limit order, if ${ }_{B}^{i} p_{t}<_{A} p_{t}^{\text {best }}$

- a market order, if ${ }_{B}^{i} p_{t} \geq_{A} p_{t}^{\text {best }}$

Limit orders can remain pending in the book for a limited time, chosen by the trader at submission. Once that period is expired, they are cancelled. Agents cannot have simultaneous active limit orders. Following the approach of Lux and Marchesi (1999, 2000), limit orders implicitly express a signal on the "market sentiment" while they remain validly pending. This induces the dynamic adjustment of the price settings rules, as above explained, by means of $\Delta n$ and $\Delta p$. In particular, the market pressure term, $\Delta n$, is computed as:

$$
\Delta n=\left\{\begin{array}{lll}
\left(n_{A} / n_{B}\right)-1 & \text { if } & n_{A}>n_{B} \\
\left(n_{B} / n_{A}\right)-1 & \text { if } & n_{B}>n_{A}
\end{array}\right.
$$

The price pressure term is computed as the absolute value of the difference between the two best quotes in the book, i.e. $\Delta p=\left.\right|_{A} p_{t}^{\text {best }}{ }_{-}{ }_{B} p_{t}^{\text {best }} \mid$. Both terms concur in the dynamical adjustment of individual price setting rules, as read in eqs.(6) and (8), at each iteration, by means of $\left[z_{1} \Delta n+z_{2} \Delta p\right]$.

Market orders are immediately executed at the best price of the counter side of the book In case the ordered quantities of both match, i.e., if $q_{t}^{B}=q_{t}^{A}$, the negotiation regulation is easily described: the bidder (respectively to the asker) obtains the due increase (respectively decrease) in the owned asset quantity and the consequent decrease (respectively increase) in the quantity of money. In case the ordered quantity is smaller than the quantity contained in the best counter-side order, the negotiation will happen all at once and will satisfy the order completely. Instead, in case the ordered quantity is larger than the quantity contained in the best counter-side order, several transactions will occur, one after the other, unless either the ordered quantity is not entirely transacted, or the maximum number of allowed counterparts that a trader is allowed to match has not been reached. Such a parameter, whose effect has been studied in Biondo (2018a), is named "order book length". In the present paper it is kept fixed to 3 and its role is illustrated in Fig.1.

\section{Trading}

Negotiations occur finally, according to priority and validity as above explained. All prices used in transactions, in order of execution, are registered and constitute the simulated time series. In all cases when a counterpart is not found, the order book does not register any transaction and the price list is not updated, as it would happen in true markets.

\subsection{Stylized Facts of Order Book Statistics}

In this section, the preliminary compliance check of the model to some of the most known stylized facts is presented. These statistical regularities, defined in Cont (2001) are comprehensively reviewed in Chakraborti et al. (2011). In particular, the simulated data will exhibit: 1- fat tails of returns distribution, 2- lack of autocorrelation of returns, 3- volatility clustering. 


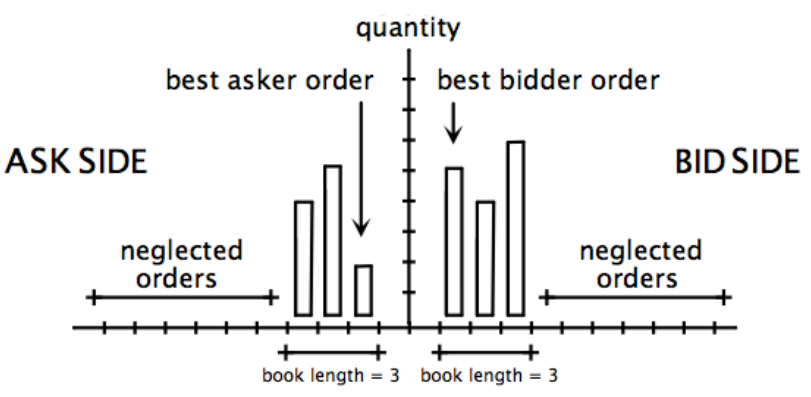

Figure 1 - Market orders negotiations.

In the depicted example, a bid market order arrives for 5 shares and the best asker is offering 2 shares. Since the order book length parameter is fixed to 3 , only three counterparts will be considered. The first transaction is negotiated between the best bidder and the best asker and executed for 2 units at the best ask. The best asker is thus erased. The matching for the remaining unsatisfied demanded quantity will be executed with the next asker, i.e., the new best asker, with her order of 5 shares at her ask price (the new best ask, which is higher than the previous one). The second transaction is executed and the market bidder is completely satisfied. Notice that, however, no more than 3 counterparts would have been matched, in any case.

Presented series have 15000 entries, net of a transient of 2000 entries. Returns have been obtained from the price series generated by the model, according to the canonical definition: $r_{t}=\left(p_{t}-p_{t-1}\right) / p_{t-1}$. The set of parameters required for the model operation actually used for the stylized-facts-compliance check are indicated in Table 1 . The initial setting for the fundamental value is $F V_{0}=50$.

The whole set of parameters has been split in two sub-groups. Each of them has been studied in a dedicated paper. The consequences deriving from different values of the first set of parameters, which are related to the composition of the population of traders (more precisely, $\sigma_{\mathbb{D}}$-governing the fundamental value variability-, $\sigma_{F}$ and $\sigma_{C}$-governing the heterogeneity degree in the population of fundamentalists and chartists, respectively-, $\sigma_{\tau}$-governing the variability of the individual length chosen for the time window used by each chartist to infer the trend), have been described in Biondo (2018a). The second set of parameters, which are related to informative dynamics and to the strength of the feedback coming from the individual perception of market dynamics, (more precisely, ${ }^{i} \mu_{\alpha}$-governing the speculative aggressiveness of traders-, ${ }^{i} \mu_{\beta}$-governing the individual sensitiveness to the external information-, and $z$ weights -governing the proportionality between the intensities of informative signals coming either from excess demand/supply or from bid/ask spread), has been studied in Biondo (2018b). In cited companion papers, many aspects have been tested, by investigating the effects caused by several parameters settings, simulating policies, with regards to: behavioral and informative heterogeneity; environmental variability of informative signals; quantity constraints; order book length; validity of orders; indolence or speculative aggressiveness of traders; taxes and transaction costs. Although a complete review of obtained results cannot be done here, for the sake of due brevity, main intuitions emerging from such a stream of research can be reported. The main rationale holds that it is possible to focus on some factors in order to dampen market volatility. More precisely, although all presented settings generate returns series replicating stylized facts of true markets, the variation of some parameters has been shown effective in fat tails reduction, i.e., in dampening market fluctuations. Thus, for example, a stabilizing policy could consist in: fostering heterogeneity of agents (in both behavior and consciousness); increasing the quality of information; reducing the time validity of limit orders; reducing 
Table 1 - Parameters setting.

\begin{tabular}{c|c|l}
\hline \hline PARAMETER & VALUE & INTERVAL LIMITS FOR: \\
\hline$\sigma_{\mathbb{D}}$ & 1 & fundamental value variability \\
$\sigma_{F}$ & 1 & fundamentalists' heterogeneity \\
$\sigma_{\tau}$ & 1 & chartists' time window length \\
$\sigma_{C}$ & 1 & chartists' heterogeneity \\
$\sigma_{\mu_{\alpha}}$ & 1 & weight of WTA/WTP estimates: \\
$\sigma_{\mu_{\beta}}$ & 1 & weight of market pressure \\
$\sigma_{z_{1}}$ & 0.9 & weight of $\Delta n$ \\
$\sigma_{z_{2}}$ & 0.7 & weight of $\Delta p$ \\
\hline \hline
\end{tabular}

the number of allowed counterparts - i.e., the penetrative capacity - to match market orders; increasing market flexibility, thus avoiding frictions, transaction costs and taxes.

The Fig. 2 shows prices and returns series generated by the model in comparison with those of four true financial assets (namely, BMW, Colgate, General Electric, and Unicredit), ranging from Jan 1st, 1973 to June 30th, 2016.
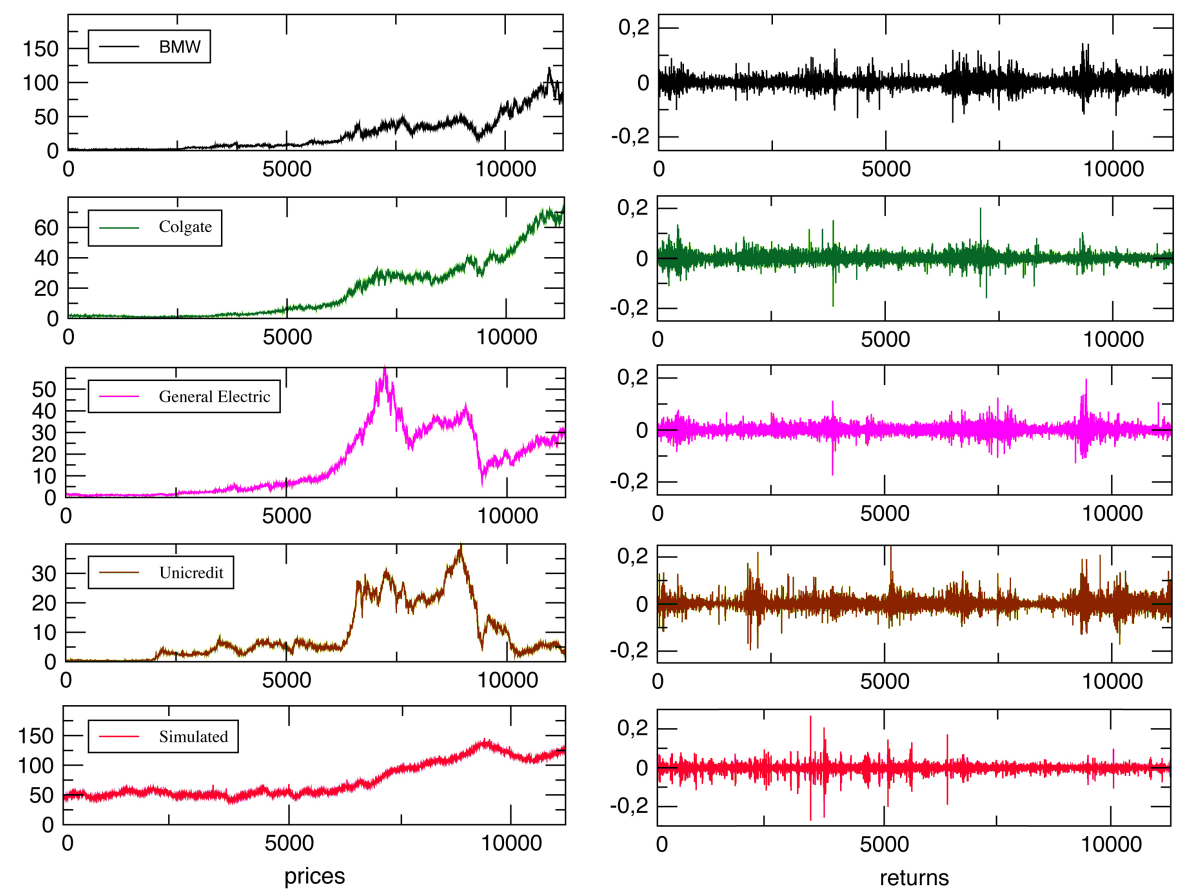

Figure 2 - Prices and Returns Comparison

First, the fat tails of the returns probability density functions has been introduced by Mandelbrot (1997), and more recently tested in Gopikrishnan et al. (1999), among others. This fact shows that the probability to find values which are distant from the average is greater than it would be in the Gaussian case. In other words, as explained in Mantegna and Stanley (1999), such a strong regularity in financial series manifest that their theoretical stochastic generator 

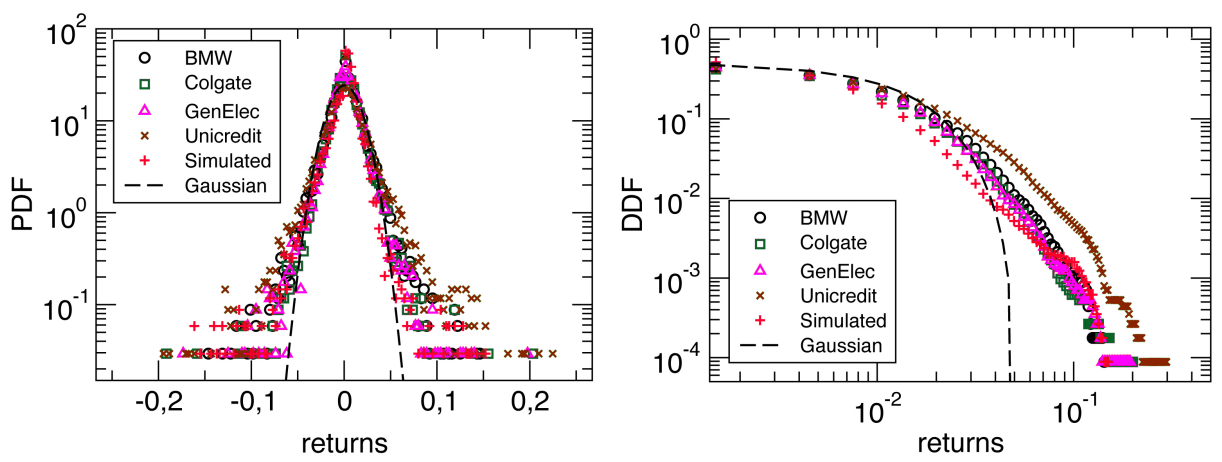

Figure 3 - Density functions of Returns Series of four true assets and of the simulated data. Left Panel: comparison of fat tails in density functions; Right Panel: Decumulative Distribution Function, defined as the probability to have a return higher of a certain value. In both panels, simulated data closely replicate the behavior of real financial data.

process would manifest infinite variance. Consequently, no predictions can be effectively done since errors in forecasts are not bounded in any standard deviation known a priori. In Fig.3, returns distributions of, respectively, the true asset returns series and the artificial price series generated by the model, are shown in log-linear plots. Simulated data exhibit a fat tailed leptokurtic PDF of returns, which very closely resembles true data.

Second, the lack of autocorrelation in the returns time series confirms the impossibility of any predictive exercise on data dynamics, as shown in Pagan (1996) and Cont et al. (1997). The autocorrelation function has been computed, as shown in the top panel of Fig.4, by reporting lag-time variation of the theoretical autocorrelation index $\rho(k)$ for up to 60 lags, in order to investigate the significance of past data in explaining the series dynamics, as:

$$
\rho(k)=\operatorname{corr}\left(r_{t}, r_{t-k}\right)=\frac{\sigma_{r_{t}} r_{t-k}}{\sigma_{r_{t}} \sigma_{r_{t-k}}}
$$

The comparison between real and simulated data shows that returns generated by the model behave well with respect to this point.

Third, the volatility clustering was firstly defined in Mandelbrot (1997). It means that periods with high volatility are followed by periods with high volatility, whereas periods with low volatility are followed by periods with low volatility. Thus, the existence of autocorrelation in absolute values of returns, shows that a non linear correlation is present. The autocorrelation function of absolute values of returns (both simulated and true) has been computed and shown in the bottom panel of Fig.4. Simulated data exhibits a slightly steeper slope, but it still appears very similar to true data: all series exhibit a positive and decreasing ACF.

\section{Micro Features and Macro Effects}

Financial volatility affects the "efficient" allocation of capitals and can become harmful for all market participants. Power law configurations of density functions of financial time series show that the unpredictability of asset prices dynamics can be hardly managed. This section highlights the effects caused by individual characteristics of agents - namely, the risk aversion and the ability to learn from past prediction errors - on the aggregate market dynamics. 


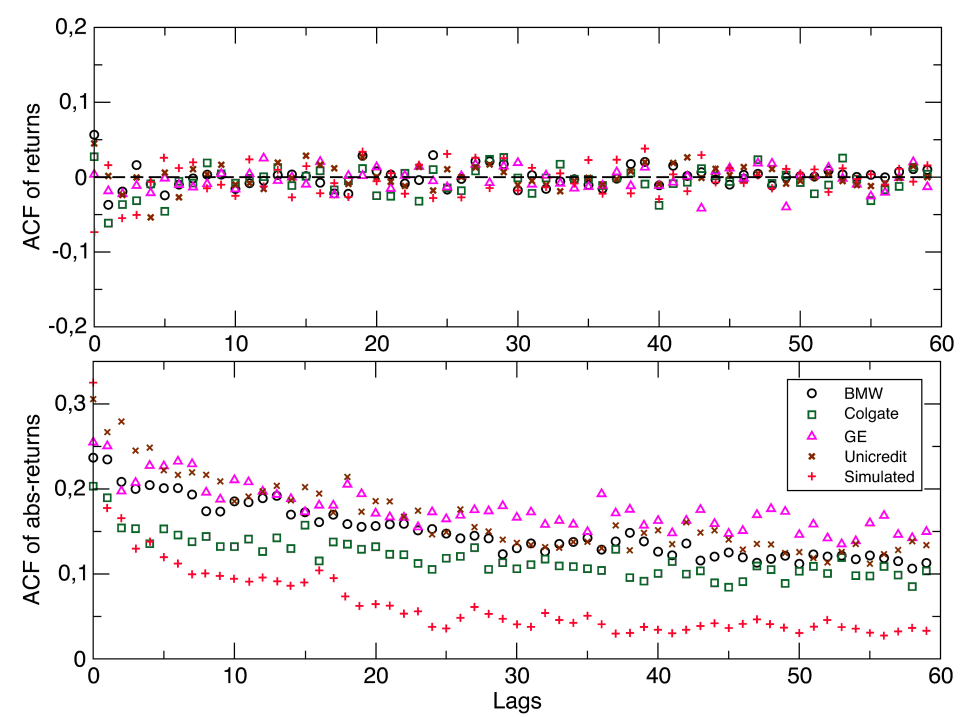

Figure 4 - Autocorrelation and Volatility Clustering. In the top panel, all series appear to be uncorrelated with themselves. In the bottom panel, the positive decaying autocorrelation of absolute values of returns, shows the volatility clustering phenomenon: high volatility is followed by high volatility; low volatility is followed by low volatility.

\subsection{Risk aversion and market dynamics}

The first feature being tested is the risk aversion of traders. The above-shown baseline model has been augmented with an individual behavioral attribute representing the risk aversion. Given that traders have not been endowed with a utility function, such a personal feature has not been treated as it usually does in related literature on the topic (see, for example, Zhou et al. (2017)). Here, agents are defined either "risk-lover/neutral" or "risk-averse", by means of a binary parameter, randomly distributed at the beginning of simulations.

In particular, in comparison with the baseline model, each agent forms two expectations, one referred to the short run, and one to the long run. The short-run expectation, $p_{S R}^{\exp }$, is calculated as the expected price defined in eqs.(2) and (4). Instead, the long-run expectation, $p_{L R}^{\exp }$, is, respectively, the fundamental value for fundamentalists, and the reference value for chartists, as defined in eqs.(1) and (3).

In this model a behavioral representation of the risk aversion is advanced. In fact, traders have not a utility function. Thus, their perception of risk does not affect the value of their happiness, nor the value of their portfolios. The point is, instead, that the individual risk attitude matters on how investors decide about the future, provided that they form expectations on the market dynamics for both a short-run and a long-run perspective. Each trader sets the strategy according to her risk aversion, by means of a cautionary cross-check on expectations referred to different temporal horizons. Table 2 shows the correspondence between personal attitudes and trading decision rules:

\section{Simulation Results}

Results show that the individual risk aversion can affect the market dynamics and, more specifically, causes more instability. Such a result is surprising to a certain extent, because one could quite naturally expect that if agent are "more scared" to invest, this should dampen 
Table 2 - Two-steps validation process for trading strategy setting

\begin{tabular}{|c|c|c|c|c|}
\hline Short-Term & Long-Term & Cross-Check & Risk Aversion & Chosen Strategy \\
\hline \multirow{6}{*}{$p_{S R}^{\exp }>p_{t}$} & $p_{L R}^{\exp } \geq p_{S R}^{\exp }$ & - & ALL & BUY \\
\hline & \multirow{5}{*}{$p_{L R}^{\exp }<p_{S R}^{\exp }$} & $p_{L R}^{\exp }>p_{t}$ & ALL & BUY \\
\hline & & \multirow{2}{*}{$p_{L R}^{\exp }=p_{t}$} & LOVER/NEUTRAL & BUY \\
\hline & & & AVERSE & HOLD \\
\hline & & \multirow{2}{*}{$p_{L R}^{\exp }<p_{t}$} & LOVER/NEUTRAL & HOLD \\
\hline & & & AVERSE & SELL \\
\hline \multirow{5}{*}{$p_{S R}^{e x p}=p_{t}$} & \multirow{2}{*}{$p_{L R}^{\exp }>p_{S R}^{\exp }$} & - & LOVER/NEUTRAL & BUY \\
\hline & & - & AVERSE & HOLD \\
\hline & $p_{L R}^{\exp }=p_{S R}^{\exp }$ & - & ALL & HOLD \\
\hline & \multirow{2}{*}{$p_{L R}^{\exp }<p_{S R}^{\exp }$} & - & LOVER/NEUTRAL & HOLD \\
\hline & & - & AVERSE & SELL \\
\hline \multirow{6}{*}{$p_{S R}^{\exp }<p_{t}$} & \multirow{5}{*}{$p_{L R}^{\exp }>p_{S R}^{\exp }$} & \multirow{2}{*}{$p_{L R}^{\exp }>p_{t}$} & LOVER/NEUTRAL & BUY \\
\hline & & & AVERSE & HOLD \\
\hline & & \multirow{2}{*}{$p_{L R}^{\exp }=p_{t}$} & LOVER/NEUTRAL & HOLD \\
\hline & & & AVERSE & SELL \\
\hline & & $p_{L R}^{\exp }<p_{t}$ & ALL & SELL \\
\hline & $p_{L R}^{\exp } \leq p_{S R}^{\exp }$ & - & ALL & SELL \\
\hline
\end{tabular}

price variability and, in turn, returns volatility. This is as to say that fat tails are expected to be reduced. Instead, results of simulations confirms the opposite view: risk aversion slightly increases market instability and fat tails of the returns PDFs results to be fatter. Fig.5 shows the increasing impact on market volatility of the percentage of risk-averse traders. In the left panel, the log-linear plot of the density function of simulated returns is depicted and it shows the leptokurtic fat-tailed distribution of returns; in the right panel, the decumulative distribution function (i.e., the probability to have a return higher of a certain value) of returns is reported. In both panels, fat tails become fatter as the percentage of risk-averse traders increases.

The rationale of such an evidence is not strange: the behavioral risk aversion here introduced, i.e. the attitude of being particularly cautious, eventually induces a trader to follow the market in just a half of the cases. In all other situations, the risk-averse agent either trades against the market or simply holds on. Therefore, compared with the baseline model, the market overreacts to trend reversion and volatility increases. Further, the double-check mechanism used to validate expectations causes a sort of fragmentation in the flexibility of market dynamics. It operates similarly to putting thresholds in decision rules and implicitly creates "classes" of traders, whose strategies are eventually triggered by common (or very similar) trading rules. This result confirms that any form of behavioral homogeneity, specially in cases when it is a widespread opinion, generates instability and fatter tails of returns PDFs, as confirmed by tests done in Biondo (2018a).

\subsection{Individual learning}

The second feature being tested is the learning ability of agents with respect to the misalignments of their past predictions with respect to occurred values. The learning to forecast 

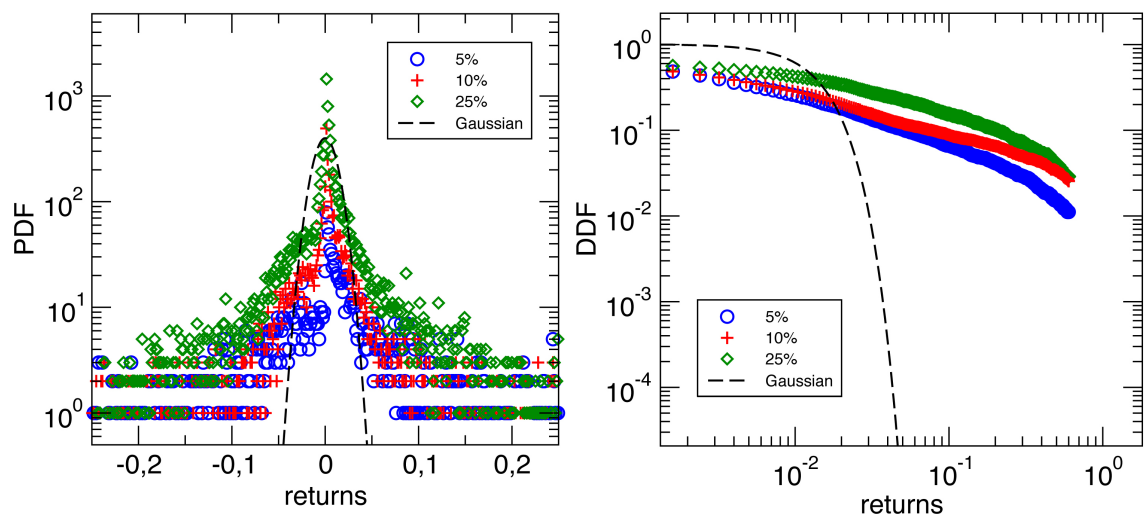

Figure 5 - Risk-aversion and market volatility

hypothesis stands for the fact that people observe realized prices and, individually, know their errors in forecasting while ignoring the history of other individuals' predictions. Thus, each trader may adopt an adjusting mechanism that considers, time after time, past errors in creating the new expectations. Here, similarly to Colasante et al. $(2017,2018)$, agents are designed to settle their expectations by means of a simplified version of the rule presented in Heemeijer et al. (2009). Specifically, each trader $i$ forms her expected price as:

$$
{ }_{j}^{i} p_{t}^{\exp { }^{L t F}}={ }_{j}^{i} p_{t}^{\exp }+\alpha\left(p_{t}-{ }_{j}^{i} p_{t-1}^{\exp }\right)+\beta\left(p_{t-1}-{ }_{j}^{i} p_{t-2}^{\exp }\right)
$$

where, ${ }_{j}^{i} p_{t}^{\text {exp }}$ is the expectation computed in $t$, according to the agent type $j=F, C$, as in eqs.(2) and (4); $L P E=\left(p_{t}-{ }_{j}^{i} p_{t-1}^{\text {exp }}\right)$ and $P P E=\left(p_{t-1}-{ }_{j}^{i} p_{t-2}^{\exp }\right)$ are the last and the past prediction errors (i.e., the misalignments between the trader's expectations and the occurred values, respectively, in $t-1$ and in $t-2$ ), and coefficients $\alpha$ and $\beta$ represent the weights of each error on future expectations.

In order to compare the efficacy of the correction mechanism, $\alpha$ and $\beta$ are defined following two different hypotheses in averaging past misalignments:

1) fixed, by considering the relevance of either a short, or a long, or both kind of memories, as shown in the following table

Table 3 - Memory length and weights of prediction errors

\begin{tabular}{lcc|cc|cc}
\hline \hline & \multicolumn{2}{c}{ SHORT } & \multicolumn{2}{c}{ LONG } & \multicolumn{2}{c}{ BOTH } \\
\cline { 2 - 7 } & $\alpha$ & $\beta$ & $\alpha$ & $\beta$ & $\alpha$ & $\beta$ \\
\cline { 2 - 7 } Scenario 1 & 0.1 & 0 & 0 & 0.1 & 0.3 & 0.5 \\
Scenario 2 & 0.3 & 0 & 0 & 0.3 & 0.5 & 0.3 \\
Scenario 3 & 0.5 & 0 & 0 & 0.5 & 0.5 & 0.5 \\
\hline \hline
\end{tabular}

2) variable, in such a way that each error counts for its relative weight with respect to a sort of measure of the total prediction errors, updated at each time step, i.e.,

$$
\alpha=\frac{|L P E|}{|L P E|+|P P E|} \quad \beta=\frac{|P P E|}{|L P E|+|P P E|}
$$



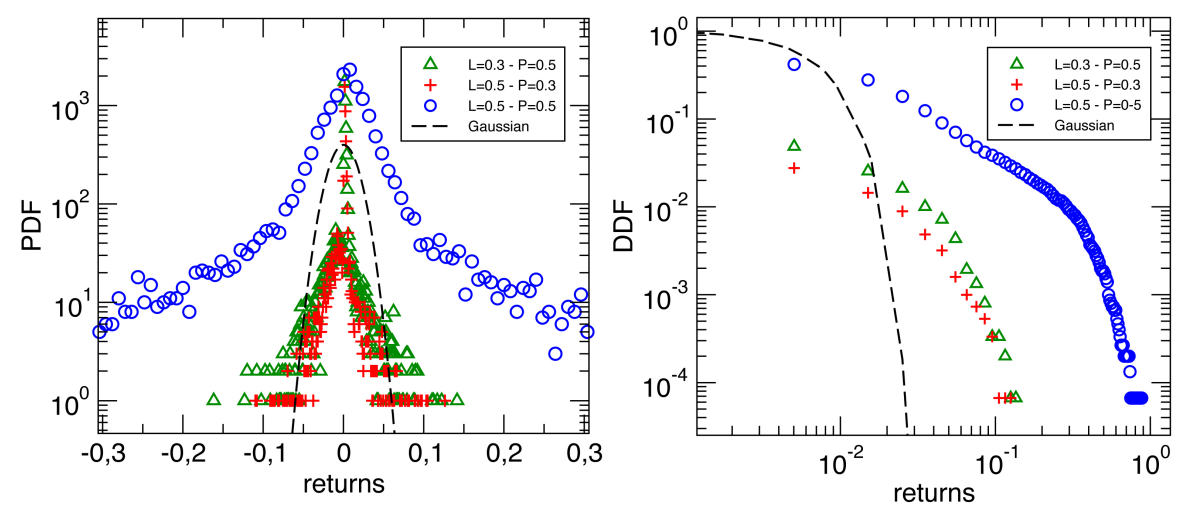

Figure 6 - Learning-to-Forecast and market stability

\section{Simulation Results}

Results show that the learning plays a role in stabilizing the market dynamics. If traders account for their errors done in past predictions when setting new expectations, the final outcome is that the market variability is dampened.

The model adopted a two-periods-based learning, in order to put an emphasis on the combined synergy of both correction mechanisms. Simulations showed that when correcting with respect both periods, best results are obtained when weights are different from each other and, in particular, when the most recent prediction errors counts more. Both panels of Fig. 6 shows exactly that when both errors are weighted the same, a cumulate and perverse destabilizing effect emerges. In the left panel, fat tails are effectively dampened when weights are asymmetric. The right panel shows that the probability to incur in more volatile fluctuations increases when both weights are set to 0.5 .

Consistently, Fig.7 reports results of simulations in which only one prediction error has been used to set expectations.

In both cases, if the used weight is too high, the use of the correction reveals to be harmful, with respect to the overall market dynamics. There is a small, though recognizable, difference in results obtained with weights set to 0.1 and 0.3 . Instead, fat tails unambiguously increase when half of the error is considered in setting new expectations. The rationale is that if agents assign too weight to past errors, they risk to amplify past dynamics as in a dangerous loop which exacerbates market instability, which resembles what happens when a microphone is put too close to a speaker.

Finally, simulation results show that the configuration with variable weights, updated at each time step, is less effective in reducing fat tails than with fixed ones. This means that the weights calculated as a relative proportion of the overall predictive inability act more dramatically on price variability, as reported in Fig.8. The left panel clearly puts in evidence that bad effect of the combined action of both corrections acts even worse than in the fixed weights case. When weights are variable, their computation reflects the relative magnitude of each error with respect to the overall misalignment, computed as in eq.(12). Such a result is consistent with above-shown Fig.6: indeed, in the variable setting, both weights can frequently be greater than 0.3 , and this ends up in fatter tails.

Moreover, since Fig. 8 has been drawn by comparing the most effective results of each test done for learning, it shows the overall effects on market dynamics of different hypotheses. 

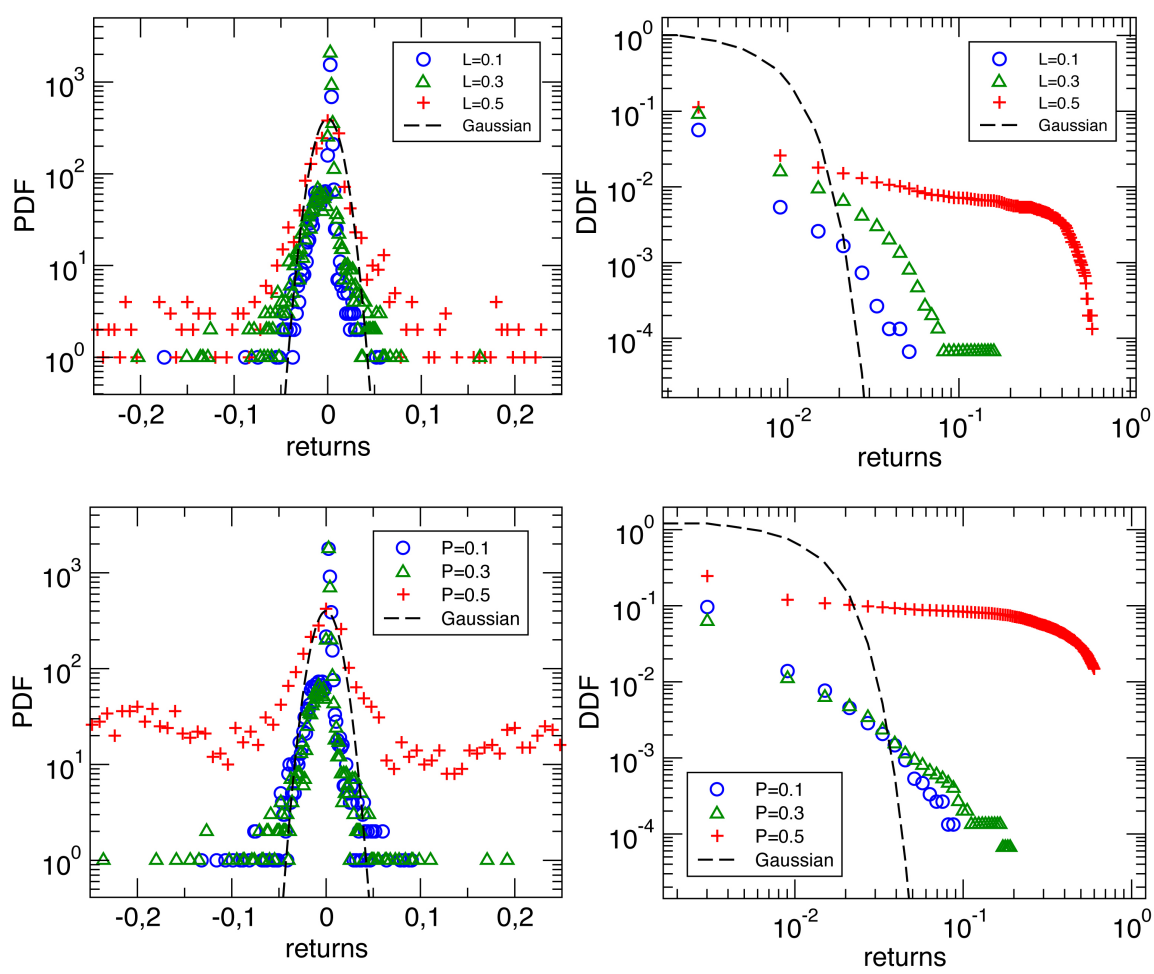

Figure 7 - Learning-to-Forecast: last vs past prediction error.

Consistently with what has been previously illustrated, when considering just one correction, LPE performs better than PPE. To consider both is better, but it reveals to be important to set a greater weight to LPE and a lower one to PPE.

\section{Conclusive Remarks}

In this paper, a new model of financial order book has been presented. A peculiar advantage of this model, compared to existing models, is that simulated data is exclusively generated by transactions among traders. In order to assess the reliability of the model, its compliance with some of the most relevant stylized facts of true financial time series has been shown.

The main discussion has been oriented to underline the role played by individual features namely the risk aversion of investors and the learning capacity from past prediction errors on the aggregate dynamics of the market. Results of simulations showed that such features cause two opposite effects.

In particular, when risk aversion is considered as a behavioral ingredient of trading decisions, it exhibited a counterintuitive effect, by exacerbating fat tails of density functions of returns. The rationale for such a surprising outcome is that the double-check mechanism adopted to simulate a more cautionary approach to trading, causes a sort of fragmentation in the flexibility of market dynamics. It operates similarly to thresholds in decision rules and implicitly creates "classes" of traders, whose strategies are triggered by common rules. This result confirms that any form of behavioral homogeneity, specially in cases when it is a 

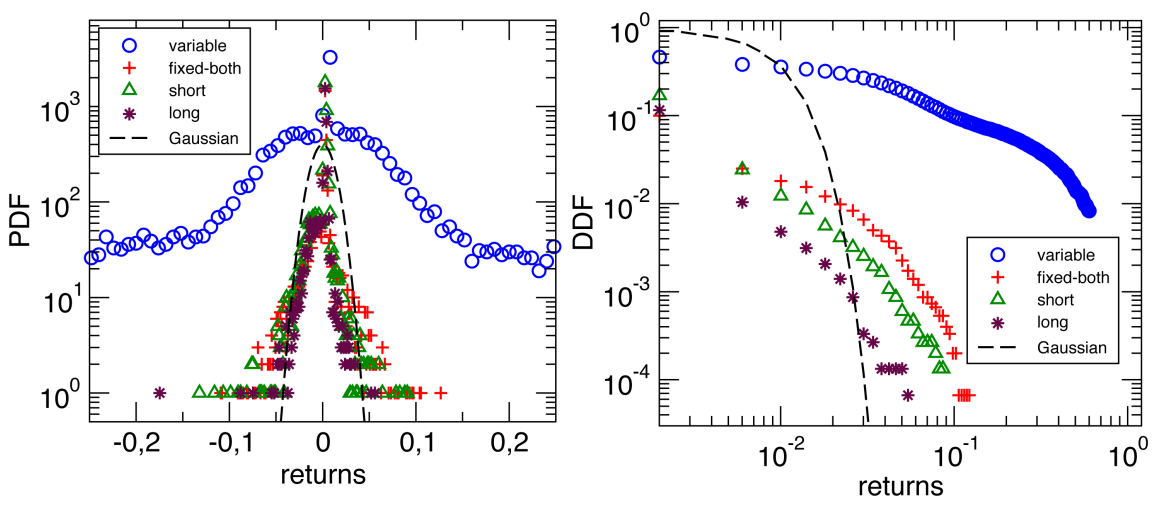

Figure 8 - Learning-to-Forecast: variable vs fixed weights.

widespread opinion, generates instability and fatter tails of returns PDFs, as confirmed by tests done in Biondo (2018a,b).

The behavioral learning attitude showed, instead, a stabilizing effect, provided that some conditions are met. When agents learn from their past errors, and adopt correction mechanisms to account for past deviations of their forecasts from correct values, market oscillations can be dampened, i.e., fat tails of returns PDFs can be reduced. The rationale of such an evidence is that, apart from the purely theoretical hypothesis of always-perfectly informed agents, an adaptive correction scheme reveals to be very useful from the aggregate point of view, as confirmed by experiments done in related literature (see Colasante et al. $(2017,2018)$ and Anufriev et al. (2013), among others).

The policy implications emerging from obtained results are oriented to favor a climate where investors can develop differentiated perceptions of risk, i.e., to reduce the credibility of informative sources about presumed levels of risk and volatility and to encourage the individual understanding of the market activity. In other words, a higher level of financial awareness of traders can be helpful in reducing waves of optimism and pessimism, which induce wide price fluctuations.

Expectations and risk perceptions must be different enough, which is as to say that herding effects in information must be fought very strongly, in order to allow a sufficiently differentiated behavior of traders and a fluid market dynamics.

\section{References}

Alfi, V., Coccetti, F., Marotta, M., Pietronero, L., and Takayasu, M. (2006). Hidden forces and fluctuations from moving averages: A test study. Physica A: Statistical Mechanics and its Applications, 370(1): 30-37. URL https://arxiv.org/abs/physics/0601089.

Alfi, V., De Martino, A., Pietronero, L., and Tedeschi, A. (2007). Detecting the traders' strategies in minority-majority games and real stock-prices. Physica A: Statistical Mechanics and its Applications, 382(1): 1-8. URL https://arxiv.org/abs/physics/0609038.

Alfi, V., Pietronero, L., and Zaccaria, A. (2008). Minimal agent based model for the origin and self-organization of stylized facts in financial markets. ArXiv preprint arXiv:0807.1888. URL https://arxiv.org/abs/0807.1888. 
Aliabadi, D. E., Kaya, M., and Sahin, G. (2017). Competition, risk and learning in electricity markets: An agent-based simulation study. Applied energy, 195: 1000-1011. URL https: //doi.org/10.1016/j.apenergy.2017.03.121.

Anufriev, M., Hommes, C., and Makarewicz, T. (2013). Learning to forecast with genetic algorithms. Discussion paper, Tech. rep.(February 2013). URL http://publish.illinois.edu/ essummer2013/files/2013/06/paper85.pdf.

Bak, P., Paczuski, M., and Shubik, M. (1997). Price variations in a stock market with many agents. Physica A: Statistical Mechanics and its Applications, 246(3-4): 430-453. URL https://doi.org/10.1016/S0378-4371(97)00401-9.

Bao, T., Hommes, C., and Makarewicz, T. (2017). Bubble formation and (In) efficient markets in learning-to-forecast and optimise experiments. The Economic Journal, 127(605). URL https://doi.org/10.1111/ecoj.12341.

Biondo, A. E. (2018a). Order book microstructure and policies for financial stability. Studies in Economics and Finance, (just-accepted): 00-00.

Biondo, A. E. (2018b). Order book modeling and financial stability. forthcoming.

Biondo, A. E., Pluchino, A., and Rapisarda, A. (2017). Informative contagion dynamics in a multilayer network model of financial markets. Italian Economic Journal, 3(3): 343-366. URL https://doi.org/10.1007/s40797-017-0052-4.

Bouchaud, J.-P., Farmer, J., and Lillo, F. (2009). How markets slowly digest changes in supply and demand. In T. Hens, and R. K. Schenk-Hoppe (Eds.), Handbook of Financial Markets. Dynamics and Evolution, volume Hens. Elsevier. URL https://arxiv.org/abs/0809.0822.

Brock, W. A., and Hommes, C. H. (1997). A rational route to randomness. Econometrica: Journal of the Econometric Society, pages 1059-1095. URL https://www.econometricsociety. org/publications/econometrica/1997/09/01/rational-route-randomness.

Brock, W. A., and Hommes, C. H. (1998). Heterogeneous beliefs and routes to chaos in a simple asset pricing model. Journal of Economic dynamics and Control, 22(8-9): 12351274. URL https://doi.org/10.1016/S0165-1889(98)00011-6.

Chakraborti, A., Toke, I. M., Patriarca, M., and Abergel, F. (2011). Econophysics review: I. Empirical facts. Quantitative Finance, 11(7): 991-1012. URL https://arxiv.org/abs/0909. 1974.

Chakravarty, S., and Holden, C. W. (1995). An integrated model of market and limit orders. Journal of Financial Intermediation, 4(3): 213-241. URL https://doi.org/10.1006/jfin.1995. 1010.

Chiarella, C. (1992). The dynamics of speculative behaviour. Annals of operations research, 37(1): 101-123. URL https://link.springer.com/article/10.1007/BF02071051.

Chiarella, C., He, X., et al. (2001). Asset price and wealth dynamics under heterogeneous expectations. Quantitative Finance, 1(5): 509-526. URL https://doi.org/10.1088/1469-7688/ $1 / 5 / 303$. 
Chiarella, C., and Iori, G. (2002). A simulation analysis of the microstructure of double auction markets*. Quantitative finance, 2(5): 346-353. URL http://www.tandfonline.com/ doi/abs/10.1088/1469-7688/2/5/303.

Chiarella, C., Iori, G., and Perelló, J. (2009). The impact of heterogeneous trading rules on the limit order book and order flows. Journal of Economic Dynamics and Control, 33(3): 525-537. URL https://arxiv.org/abs/0711.3581.

Colasante, A., Alfarano, S., Camacho, E., and Gallegati, M. (2018). Long-run expectations in a learning-to-forecast experiment. Applied Economics Letters, 25(10): 681-687. URL https://doi.org/10.1080/13504851.2017.1355537.

Colasante, A., Palestrini, A., Russo, A., and Gallegati, M. (2015). Heterogeneous adaptive expectations and coordination in a learning-to-forecast experiment. MPRA paper 66578. URL https://www.researchgate.net/publication/281772679_Heterogeneous_ Adaptive_Expectations_and_Coordination_in_a_Learning-to-Forecast_Experiment.

Colasante, A., Palestrini, A., Russo, A., and Gallegati, M. (2017). Adaptive expectations versus rational expectations: Evidence from the lab. International Journal of Forecasting, 33(4): 988-1006. URL https://doi.org/10.1016/j.ijforecast.2017.06.003.

Consiglio, A., Lacagnina, V., and Russino, A. (2005). A simulation analysis of the microstructure of an order driven financial market with multiple securities and portfolio choices. Quantitative Finance, 5(1): 71-87. URL https://doi.org/10.1080/14697680500041437.

Cont, R. (2001). Empirical properties of asset returns: stylized facts and statistical issues. Quantitative Finance, 1: 1-14. URL http://citeseer.ist.psu.edu/viewdoc/summary?doi=10.1. 1.16.5992.

Cont, R., Potters, M., and Bouchaud, J.-P. (1997). Scaling in stock market data: stable laws and beyond. In Scale invariance and beyond, pages 75-85. Springer. URL https: //arxiv.org/abs/cond-mat/9705087.

Cont, R., Stoikov, S., and Talreja, R. (2010). A stochastic model for order book dynamics. Operations research, 58(3): 549-563. URL http://www.jstor.org/stable/40792679.

Copeland, T. E., and Galai, D. (1983). Information effects on the bid-ask spread. The Journal of Finance, 38(5): 1457-1469. URL http://www.jstor.org/stable/2327580.

Daniels, M. G., Farmer, J. D., Gillemot, L., Iori, G., and Smith, E. (2003). Quantitative model of price diffusion and market friction based on trading as a mechanistic random process. Physical review letters, 90(10): 108102. URL https://www.ncbi.nlm.nih.gov/ pubmed/12689037.

Day, R. H., and Huang, W. (1990). Bulls, bears and market sheep. Journal of Economic Behavior \&amp; Organization, 14(3): 299-329. URL https://doi.org/10.1016/0167-2681(90) 90061-H.

Epstein, J. M. (2002). Modeling civil violence: An agent-based computational approach. Proceedings of the National Academy of Sciences, 99(suppl 3): 7243-7250. URL http: //www.pnas.org/content/99/suppl_3/7243.full. 
Fabretti, A., and Herzel, S. (2017). An agent based model for a double auction with convex incentives. Journal of Artificial Societies and Social Simulation, 20(1). URL http://jasss.soc. surrey.ac.uk/20/1/7.html.

Farmer, J. D., Patelli, P., and Zovko, I. I. (2005). The predictive power of zero intelligence in financial markets. Proceedings of the national academy of sciences of the united states of america, 102(6): 2254-2259. URL http://www.pnas.org/content/102/6/2254.full.

Foucault, T. (1999). Order flow composition and trading costs in a dynamic limit order market1. Journal of Financial markets, 2(2): 99-134. URL https://doi.org/10.1016/S1386-4181(98) 00012-3.

Franke, R., and Sethi, R. (1998). Cautious trend-seeking and complex asset price dynamics. Research in Economics, 52(1): 61-79. URL https://doi.org/10.1006/reec.1997.0157.

Gallegati, M., and Richiardi, M. G. (2009). Agent based models in economics and complexity. In Complex Systems in Finance and Econometrics, pages 3053. Springer. URL https://www.researchgate.net/publication/228591174_Agent_Based_ Models_in_Economics_and_Complexity.

Gatti, D. D., Desiderio, S., Gaffeo, E., Cirillo, P., and Gallegati, M. (2011). Macroeconomics from the Bottom-up, volume 1. Springer Science \&amp; Business Media.

Gil-Bazo, J., Moreno, D., and Tapia, M. (2007). Price dynamics, informational efficiency, and wealth distribution in continuous double - auction markets. Computational Intelligence, 23(2): 176-196. URL http://onlinelibrary.wiley.com/doi/10.1111/j.1467-8640.2007.00301. $\mathrm{x} / \mathrm{abstract}$.

Glosten, L. R. (1994). Is the electronic open limit order book inevitable? The Journal of Finance, 49(4): 1127-1161. URL https://www0.gsb.columbia.edu/mygsb/faculty/research/ pubfiles/1537/is.pdf.

Glosten, L. R., and Milgrom, P. R. (1985). Bid, ask and transaction prices in a specialist market with heterogeneously informed traders. Journal of Financial Economics, 14(1): 71-100. URL https://doi.org/10.1016/0304-405X(85)90044-3.

Gopikrishnan, P., Plerou, V., Amaral, L. A. N., Meyer, M., and Stanley, H. E. (1999). Scaling of the distribution of fluctuations of financial market indices. Physical Review E, 60(5): 5305. URL https://www.ncbi.nlm.nih.gov/pubmed/11970400.

Haer, T., Botzen, W., Moel, H., and Aerts, J. C. (2017). Integrating household risk mitigation behavior in flood risk analysis: An agent-based model approach. Risk Analysis, 37(10): 1977-1992. URL http://onlinelibrary.wiley.com/doi/10.1111/risa.12740/abstract.

Heemeijer, P., Hommes, C., Sonnemans, J., and Tuinstra, J. (2009). Price stability and volatility in markets with positive and negative expectations feedback: An experimental investigation. Journal of Economic dynamics and control, 33(5): 1052-1072. URL https: //doi.org/10.1016/j.jedc.2008.09.009.

Hollifield, B., Miller, R. A., and Sandås, P. (2004). Empirical analysis of limit order markets. The Review of Economic Studies, 71(4): 1027-1063. URL http://www.jstor.org/stable/ 3700727. 
Hollifield, B., Miller, R. A., Sandås, P., and Slive, J. (2006). Estimating the gains from trade in limit-order markets. The Journal of Finance, 61(6): 2753-2804. URL http: //onlinelibrary.wiley.com/doi/10.1111/j.1540-6261.2006.01004.x/abstract.

Hommes, C., and Lux, T. (2013). Individual expectations and aggregate behavior in learningto-forecast experiments. Macroeconomic Dynamics, 17(2): 373-401. URL https://doi.org/ 10.1017/S1365100511000162.

Hommes, C. H. (2001). Financial markets as nonlinear adaptive evolutionary systems. URL https://doi.org/10.1080/713665542.

Hommes, C. H. (2006). Heterogeneous agent models in economics and finance. In L. Tesfatsion, and K. L. Judd (Eds.), Handbook of Computational Economics, , volume 2, pages 1109-1186. North-Holland.

Kirman, A. (2010). Complex economics: individual and collective rationality. Routledge.

Kyle, A. S. (1985). Continuous auctions and insider trading. Econometrica: Journal of the Econometric Society, pages 1315-1335. URL http://www.jstor.org/stable/1913210.

Ladyman, J., Lambert, J., and Wiesner, K. (2013). What is a complex system? European Journal for Philosophy of Science, 3(1): 33-67. URL https://link.springer.com/article/10. 1007/s13194-012-0056-8.

LeBaron, B. (2006). Agent-based computational finance. In J. K. L. e. Tesfatsion, Leigh (Ed.), Handbook of Computational Economics, volume 2, pages 1187-1233. North-Holland. URL https://doi.org/10.1016/S1574-0021(05)02024-1.

Lux, T. (1995). Herd behaviour, bubbles and crashes. The economic journal, pages 881-896. URL ttp://www.jstor.org/stable/2235156.

Lux, T. (1998). The socio-economic dynamics of speculative markets: interacting agents, chaos, and the fat tails of return distributions. Journal of Economic Behavior \&amp; Organization, 33(2): 143-165. URL https://doi.org/10.1016/S0167-2681(97)00088-7.

Lux, T., and Marchesi, M. (1999). Scaling and criticality in a stochastic multi-agent model of a financial market. Nature, 397(6719): 498. URL https://www.nature.com/articles/17290.

Lux, T., and Marchesi, M. (2000). Volatility clustering in financial markets: a microsimulation of interacting agents. International journal of theoretical and applied finance, 3(04): 675702. URL https://www.researchgate.net/publication/45122249_Volatility_Clustering_in_ Financial_Markets_A_MicroSimulation_of_Interacting_Agents.

Mandelbrot, B. B. (1997). The variation of certain speculative prices. In Fractals and scaling in finance, pages 371-418. Springer. URL http://www.jstor.org/stable/2350970.

Mantegna, R. N., and Stanley, H. E. (1999). Introduction to econophysics: correlations and complexity in finance. Cambridge university press.

Maslov, S. (2000). Simple model of a limit order-driven market. Physica A: Statistical Mechanics and its Applications, 278(3-4): 571-578. URL https://arxiv.org/abs/cond-mat/ 9910502. 
Mitchell, M. (2009). Complexity: A guided tour. Oxford University Press.

Pagan, A. (1996). The econometrics of financial markets. Journal of empirical finance, 3(1): 15-102. URL https://doi.org/10.1016/0927-5398(95)00020-8.

Parlour, C. A. (1998). Price dynamics in limit order markets. The Review of Financial Studies, 11(4): 789-816. URL https://doi.org/10.1093/rfs/11.4.789.

Parlour, C. A., and Seppi, D. J. (2008). Limit order markets: A survey. In A. Thakor, and A. Boot (Eds.), Handbook of Financial Intermediation and Banking, volume 5, pages 63-95. Elsevier. URL https://www.researchgate.net/publication/253213802_Limit_Order_ Markets_A_survey.

Prigogine, I. (1997). The end of certainty the free press. New York.

Raberto, M., Cincotti, S., Focardi, S. M., and Marchesi, M. (2001). Agent-based simulation of a financial market. Physica A: Statistical Mechanics and its Applications, 299(1-2): 319-327. URL https://doi.org/10.1016/S0378-4371(01)00312-0.

Roşu, I. (2009). A dynamic model of the limit order book. The Review of Financial Studies, 22(11): 4601-4641. URL https://papers.ssrn.com/sol3/papers.cfm?abstract_id=710841.

Rosu, I. (2016). Liquidity and information in order driven markets. Working paper, SSRN eLibrary. URL http://dx.doi.org/10.2139/ssrn.1286193.

Silvestre, J. (2017). Sovereign default contagion: an agent-based model approach. URL http://pascal.iseg.utl.pt/ depeco/wp/wp082017.pdf.

Slanina, F. (2008). Critical comparison of several order-book models for stock-market fluctuations. The European Physical Journal B, 61(2): 225-240. URL https://arxiv.org/abs/ 0801.0631.

Takayasu, M., Mizuno, T., and Takayasu, H. (2006). Potential force observed in market dynamics. Physica A: Statistical Mechanics and its Applications, 370(1): 91-97. URL https://doi.org/10.1016/j.physa.2006.04.041.

Tedeschi, G., Iori, G., and Gallegati, M. (2012). Herding effects in order driven markets: The rise and fall of gurus. Journal of Economic Behavior \&amp; Organization, 81(1): 82-96. URL https://doi.org/10.1016/j.jebo.2011.09.006.

Tesfatsion, L. (2006). Agent-based computational economics: A constructive approach to economic theory. In L. Tesfatsion, and K. L. Judd (Eds.), Handbook of Computational Economics, volume 2, pages 831-880. North-Holland.

Tirole, J. (2015). Country solidarity in sovereign crises. American Economic Review, 105(8): 2333-63. URL https://www.aeaweb.org/articles?id=10.1257/aer.20121248.

Zhou, J., Liu, Y., Zhang, X., Gu, X., and Wang, D. (2017). Uncertain risk aversion. Journal of Intelligent Manufacturing, 28(3): 615-624. URL https://doi.org/10.1007/ s10845-014-1013-5. 


\section{Economics}

Please note:

You are most sincerely encouraged to participate in the open assessment of this article. You can do so by either recommending the article or by posting your comments.

Please go to:

http://dx.doi.org/10.5018/economics-ejournal.ja.2018-20

The Editor 